\title{
Transcatheter mitral valve replacement with Tendyne valve: The first two cases in Slovakia
}

\author{
Hulman M, Bena M, Gasparovic I, Ftacnikova A, Artemiou P \\ Medical Faculty of the Comenius University, National Institute for Cardiovascular Diseases, Clinic of Cardiac Surgery, \\ Bratislava, Slovakia
}

\begin{abstract}
Hulman M, Bena M, Gasparovic I, Ftacnikova A, Artemiou P. Transcatheter mitral valve replacement with Tendyne valve: The first two cases in Slovakia Cardiology Lett. 2021;30(1):53-56

Transcatheter mitral valve replacement (TMVR) with the Tendyne mitral valve has emerged as a novel potential therapy for patients with severe mitral valve disease who are unsuitable candidates for conventional surgery and transcatheter mitral repair with MitraClip. In this communication we present the first two cases that were performed in Slovakia and the V4 countries. Two surgically high risk female patients 78 and 79 years old respectively (EuroScore II $7.8 \%$ and $7.4 \%$ respectively) also unsuitable candidates for transcatheter mitral repair with MitraClip, underwent transapical TMVR with the Tendyne valve. Both procedures were successful: one of the patients two months after the procedure is doing well, the other patient had a complicated postoperative course and died on postoperative day 4. Moreover we present a brief description of the valve, and of the deployment technique, and an overview of the major clinical studies. Fig. 3, Ref. 7, on-line full text (Free, PDF) www.cardiologyletters.sk

Key words: mitral valve regurgitation - transcatheter mitral valve replacement - Tendyne valve prosthesis
\end{abstract}

Mitral valve regurgitation (MR) is a very common occurring valvular disease in developed countries, with approximately 3.5 million people in the United States having above moderate-to severe MR (1). Surgical repair or replacement is the gold standard therapy for patients with symptomatic MR, whereas transcatheter edge-to-edge repair with MitraClip (Abbott Structural, Santa Clara, California) has proved of clinical benefit in selected patients (2).

This report presents our initial experience which is the first in V4 countries, and the potential role for transcatheter MV replacement (TMVR) with the Tendyne valve (Abbott Structural, Santa Clara, California) for patients with severe MV disease who are unsuitable candidates for conventional surgery and transcatheter mitral repair with MitraClip.

\section{The Tendyne valve prosthesis}

The tendyne valve is a self-expanding, nitinol prosthesis with a double-frame design that contains a trileaflet porcine

From Medical Faculty of the Comenius University, National Institute for Cardiovascular Diseases, Clinic of Cardiac Surgery, Bratislava, Slovakia Manuscript received 25 January 2021; accepted for publication 2 February 2021 pericardial valve and has an effective orifice area of greater than $3.2 \mathrm{~cm}^{2}$ (standard size) or $2.0 \mathrm{~cm}^{2}$ (low profile size). The prosthesis is anatomically shaped for the mitral annulus. The outer frame contains a cuff that extends above the plane of the annulus to abut the anterior atrial wall and aortic-mitral continuity for the purposes of preventing diastolic paravalvular MR. The implanted valve is selected to be larger than the native mitral orifice. The prosthesis, which is delivered via a $34 \mathrm{~F}-36 \mathrm{~F}$ transapical sheath, is uniquely anchored with a tether connected to an epicardial hemostatic pad at the apex (Figure 1A, 1B). The Tendyne prosthesis is retrievable and repositionable after full deployment, an ability that minimizes the need for bailout open surgery (3).

\section{Technique for deployment}

The deployment procedure is performed under general anesthesia using a transapical approach via a left anterolateral minithoracotomy. A pigtail catheter is inserted through the

Address for correspondence: MUDr. Ivo Gasparovic, Medical Faculty of the Comenius University, National Institute for Cardiovascular Diseases, Pod Krasnou horkou 1, 83348 Bratislava, Slovakia, e-mail: ivo.gasparovic@nusch.sk 

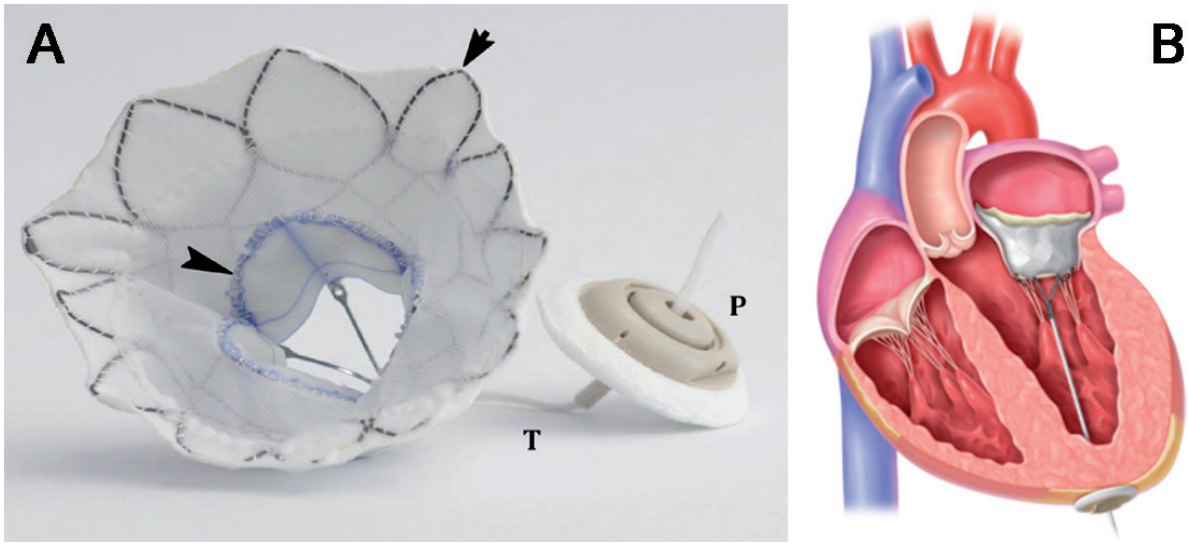

Figure 1A The Tendyne mitral valve system. The nitinol self-expanding prosthesis has a double flame. An outer flame (arrow) contains a cuff and an inner flame has a trileaflet porcine pericardial valve (arrowhead). The prosthesis is anchored to an epicardial hemostatic pad $(\mathrm{P})$ by a tether $(\mathrm{T})$

Figure 1B The Tendyne mitral valve system in place at the end of the procedure (Courtesy of Abbot, Inc, Santa Clara, CA)

femoral artery to the left ventricle, a myocardial access site is punctured with a multipurpose needle, a balloon-tip-catheter is advanced from the left ventricle to the left atrium and a $34 \mathrm{~F}-36 \mathrm{~F}$ delivery sheath is inserted into the left ventricle. The valve is deployed intrannularly under the guidance of transesophageal echocardiography and fluoroscopy. After deployment, anchoring is achieved by a tether connecting to an epicardial pad, which also provides hemostasis. The tension and length from the valve to the ventricular apex of the tether are adjusted after deployment to optimize the seating of the prosthesis without worsening of left ventricular filling pressures. If the function of the prosthesis is not acceptable or if LVOT obstruction occurs, the prosthesis can be repositioned or fully retrieved. The entire procedure is performed without cardiopulmonary bypass and without rapid ventricular pacing. Antiplatelet therapy with aspirin $100 \mathrm{mg}$ or clopidogrel $75 \mathrm{mg}$ and anticoagulant therapy with heparin followed by warfarin for greater than or equal to 3 months, with target international normalization ratio of 2.5 to 3.5 , are used in general.

\section{Patient selection}

Patient evaluation by a multidisciplinary heart team is paramount to optimize procedural results and effectiveness of TMVR. Two surgically high risk (EuroScore II $7.8 \%$ and $7.4 \%$ respectively) and unsuitable candidates for transcatheter mitral repair with MitraClip female patients, 78 and 79 years old respectively, were indicated by our institutional multidisciplinary heart team for TMVR with the Tendyne valve.

Both patients underwent preprocedural multimodality imaging with transthoracic echocardiography, transesopha- geal echocardiography (TEE) and contrast-enhanced, gated- cardiac CT.

The procedures were performed in November 2020 under general anesthesia, using a transapical approach via a left anterolateral minithoracotomy without cardiopulmonary bypass and rapid ventricular pacing. Procedural success was $100 \%$, with no or minimal paravulvular leak and without LVOT obstruction. The Tendyne bioprosthetic valves of 29 L LP and 35 M LP sizes were implanted. The first patient had a complicated postoperative course, where on the second postoperative day (POD) she underwent implantation of a peripheral venoarterial membrane oxygenation (VA-ECMO) due to cardiogenic shock. Later on POD 4 the patient died as a result of bleeding complications. The second patient had an uneventful postoperative course, she has long-term anticoagulation treatment with warfarin, and more than two months after the procedure is doing well (Figure 2A, 2B, 3A, 3B).

\section{Clinical outcomes}

The Global Feasibility Study (4) was a prospective, open-label, non-randomized trial to establish the safety and efficacy of the Tendyne valve at 30-day follow-up. A total of 30 patients with grade 3 or grade 4 and mean Society of Thoracic Surgeons (STS) predicted mortality $7.3 \% \pm 5.7 \%$ underwent TMVR using the Tendyne valve. Device implantation was successful in 28 of the 30 patients without procedural death or complications. The 30 -day survival was $86.7 \%$ (26/30 patients).

Sorajja et al. (5) reported long-term results from the first 100 patients with mean STS predicted risk of operative mortality $7.9 \% \pm 5.7 \%$ enrolled in the Global Feasibility Study. Successful valve implantation occurred in $97 \%$ of patients 

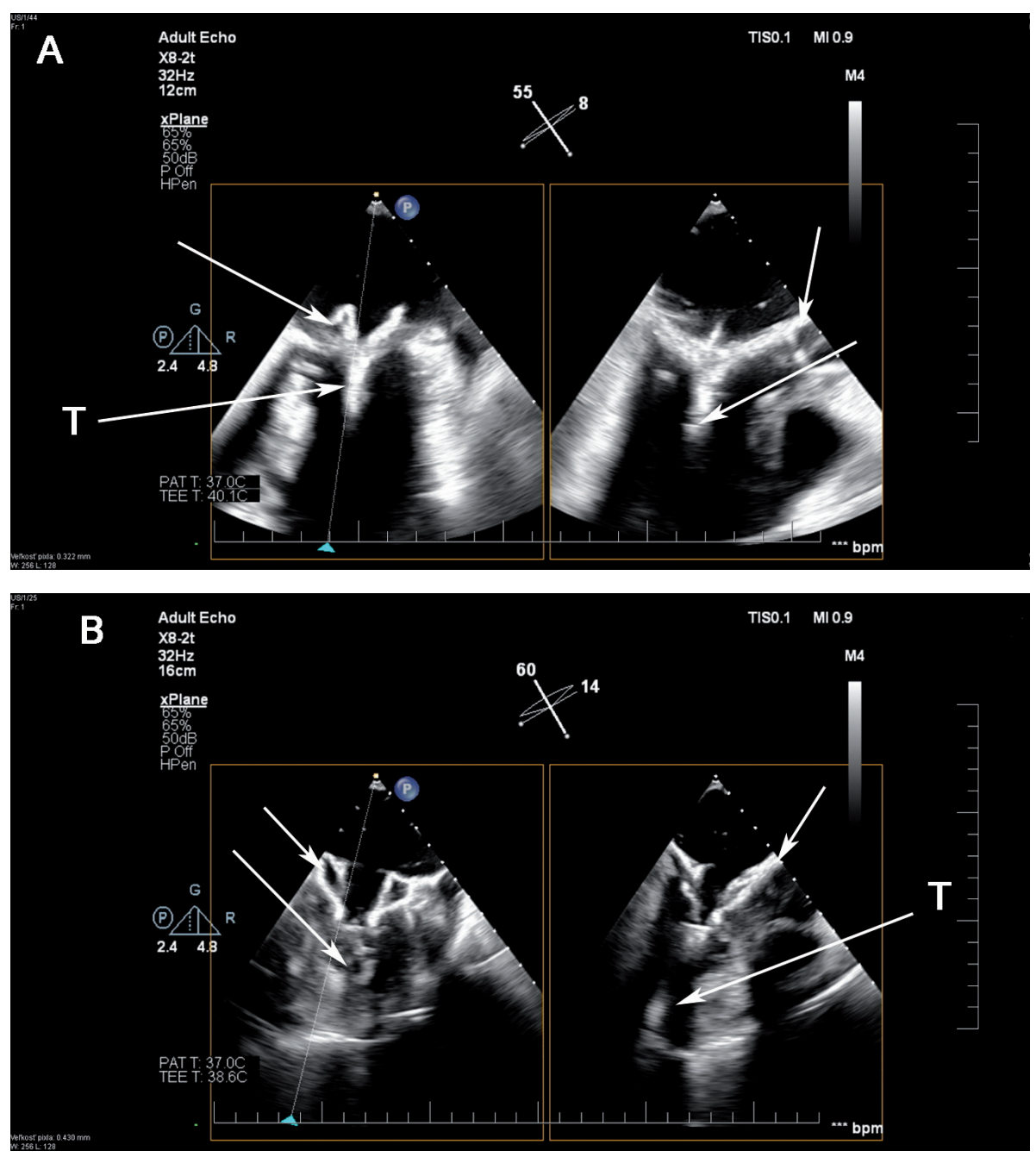

Figure 2A, 2B Intraoperative transesophageal images showing the Tendyne mitral valve prosthesis (arrows) $\mathrm{T}-$ tether

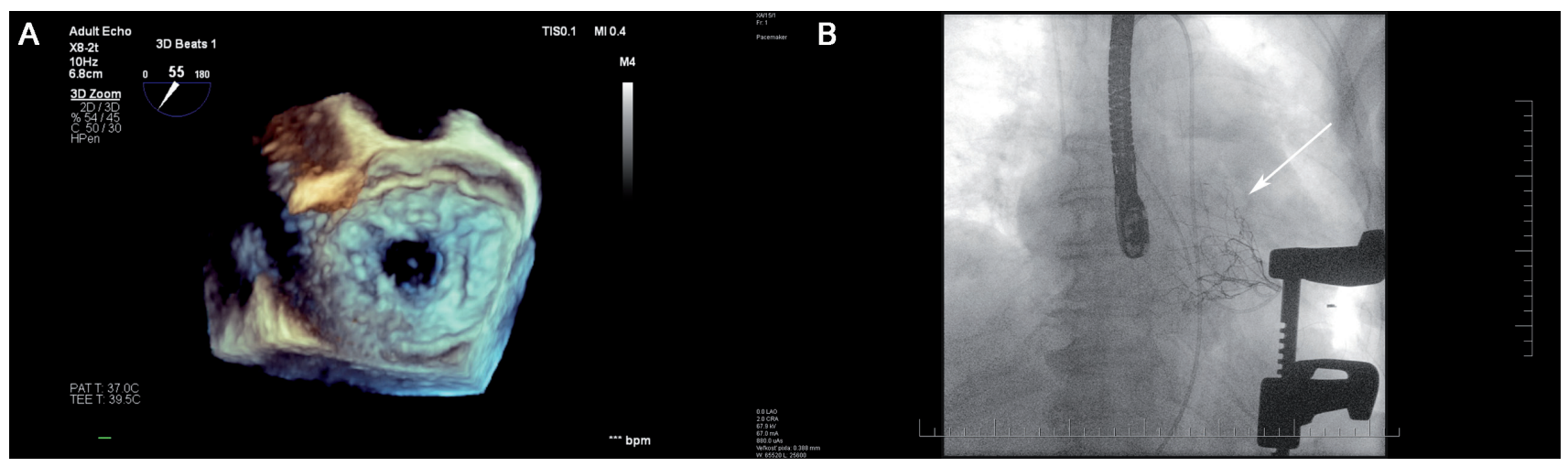

Figure 3A Intraoperative transesophageal 3D image showing the Tendyne mitral valve prosthesis fully extruded Figure 3B Intraoperative fluoroscopy demonstrating placement of the Tendyne mitral valve prosthesis (arrow) 
without procedural death or complications. The 30-day mortality was $6 \%$ and the rate of 1-year survival was $72 \%$ and $78 \%$ of the survivors had improved quality of life.

The SUMMIT (Tendyne Mitral Valve System for the Treatment of Symptomatic Mitral Regurgitation) trial (6) is a randomized 2:1 observational clinical trial that evaluates the safety and effectiveness of using the Tendyne mitral valve. The trial will enrol up to 1010 patients at 80 sites in the United States, European Union, and Canada. The trial design has a surgical (conventional surgery) and a nonsurgical arm (patients ineligible for MitraClip). The primary endpoint in both cohorts is a composite end point of death, cardiovascular hospitalization, stroke, or reoperation at 1 year.

The Tendyne mitral annular calcification (MAC) study is a feasibility study of the Tendyne valve use in patients with symptomatic MR and severe MAC not suitable for conventional surgery, which is currently recruiting patients. It is a single arm multicenter study that plans to include up to 30 patients at no more than 10 centers. The primary endpoint is procedural success and procedure-related adverse events at 30-day follow-up (7).

In conclusion, TMVR has emerged as a novel potential therapy for patients with severe mitral valve disease who are unsuitable candidates for conventional surgery and transcatheter mitral repair with MitraClip, and has shown potential as an effective and safe treatment alternative for high-risk patients at short-term follow-up.

The authors declare no funding and no conflict of interest.

\section{References}

1. Nkomo VT, Gardin JM, Skelton TN, et al. Burden of valvular heart disease: a population-based study. Lancet 2006:368:1005-1011.

2. Liska B, Pacak J, Postulka J, et al. Secondary mitral regurgitation and chronic heart failure. Cardiology Lett 2020:29:34-43.

3. Niikura $H$, Gossl M, Sorajja P. Transcatheter mitral valve replacement with Tendyne. Intervent Cardiol Clin 2019:295-300.

4. Muller DW, Farivar RS, Jansz P, et al. Transcatheter mitral valve replacement for patients with symptomatic mitral regurgitation: a global feasibility trial. J Am Coll Cardiol 2017:69:381-391.

5. Sorajja P, Moat $\mathrm{N}, \mathrm{Badhwar} \mathrm{V}$, et al. Initial feasibility study of a new transcatheter mitral prosthesis. The first 100 patients. J Am Coll Cardiol 2019:73:1250-1260.

6. Clinical Trial to Evaluate the Safety and Effectivness of using the Tendyne Mitral Valve System for the Treatment of Symptomatic Mitral Regurgitation (SUMMIT) ClinicalTrials.gov.identifier, NCT03433274

7. Feasibility study of the Tendyne Mitral Valve System for Use in Subjects With Mitral Annular Calcification ClinicalTrials.gov. identifier, NCT03539458 